\title{
Tratamiento quirúrgico de una hiperplasia fibrosa localizada en la desembocadura del conducto de Estenon. Descripción de la técnica
}

\author{
Camacho Alonso $\mathrm{F}^{*}$, Saura Inglés $\mathrm{A}^{* *}$, López Jornet P***, Bermejo Fenoll A*****
}

\section{RESUMEN}

La hiperplasia fibrosa es una lesión exofítica circunscrita y formada por tejido conectivo fibroso denso, cubierto por un epitelio escamoso estratificado. Se localiza con más frecuencia en la mucosa yugal, encía, lengua y paladar blando, generalmente zonas donde se produce mayor roce, ya que su etiopatogenia parece estar relacionada con factores irritativos crónicos o traumáticos repetidos. El tratamiento consiste en la extirpación quirúrgica y la eliminación de los factores irritantes locales.

Presentamos un caso de tumoración unilateral de aspecto fibroso de diez años de evolución localizada en mucosa yugal izquierda, que incluía la carúncula de desembocadura del conducto de Estenon. El tratamiento consistió en la canalización del conducto para preservar su integridad, extirpación quirúrgica de la lesión con bisturí frío y colocación de un drenaje activo intraoral. El estudio histopatológico de la muestra confirmó el diagnóstico de hiperplasia fibrosa. Tras un año de seguimiento, el paciente no ha presentado ninguna recidiva, y el drenaje de la glándula parótida izquierda se mantiene normal.

Palabras clave: Hiperplasia fibrosa, conducto de Estenon, glándula parótida.

\begin{abstract}
The fibrous hyperplasia is an exofitic injury circumscribed and formed by dense fibrous conective weave, covered by epithelio squamous stratified. It is located with more frequency in the buccal mucosa, gingiva, tongue and soft palate, generally zones where greater rubbing takes place, since his etiopathogenia seems to be related to chronic irritatives or traumatic repeated factors. The treatment is based in the surgical extirpation and the elimination of the local irritating factors.

We present a case of unilateral tumor of fibrous aspect of ten years of evolution located in left buccal mucosa, that included caruncula of opening of Estenon's duct. The treatment consisted of the canalization of the duct to preserve its integrity, surgical extirpation of the injury with cold scalpel and positioning of an active intraoral drainage. The histopathologic study of the sample confirmed the diagnosis of fibrous hyperplasia. After one year follow-up, the patient has not presented any recurrence, and the drainage of the left parotid gland is functioning normally.

Key words: Fibrous hyperplasia, Stensen's duct, parotid gland.

* Máster de Cirugía e Implantología Bucal por la Facultad de Medicina y Odontología de la Universidad de Valencia. Profesor colaborador de Medicina Bucal. Facultad de Medicina y Odontología. Universidad de Murcia.

** Profesor Asociado de Medicina Bucal. Facultad de Medicina y Odontología. Universidad de Murcia.

*** Profesora titular de Medicina Bucal. Facultad de Medicina y Odontología. Universidad de Murcia.

***** Catedrático de Medicina Bucal. Facultad de Medicina y Odontología. Universidad de Murcia.
\end{abstract}


Camacho Alonso F, Saura Inglés A, López Jornet P, Bermejo Fenoll A. Tratamiento quirúrgico de una hiperplasia fibrosa localizada en la desembocadura del conducto de Estenon. Descripción de la técnica. Au. Odontoestomatol 2004; 20-5: 227-231.

\section{INTRODUCCIÓN}

El nódulo fibroso o hiperplasia fibrosa es la lesión exofítica más frecuente de la cavidad oral. En muchas ocasiones indistinguible del fibroma. Representa casi el $20 \%$ de las lesiones encontradas en la mucosa bucal. Es más frecuente en mujeres que en hombres y la mayor prevalencia se halla alrededor de la tercera década de la vida (1). Su etiología parece estar relacionada con factores irritativos crónicos o traumáticos repetidos tales como malos hábitos, prótesis y aparatos ortodóncicos. También se le atribuye una etiología por succión de la mucosa (en diastemas) que tiende a ocupar los espacios vacíos. Puede localizarse en cualquier zona de la mucosa oral aunque con más predilección por las regiones donde se produce mayor roce: mucosa yugal, encía, lengua y paladar blando.

Clínicamente aparece como una lesión generalmente única, bien definida, de crecimiento lento y autolimitado. Suele ser asintomática, de aspecto nodular, elevada, pedunculada y sésil, de tamaño variable. El color de la mucosa que la cubre es el de la mucosa normal aunque ésta puede ser más pálida, tersa, lisa y brillante. Su consistencia puede variar desde firme y resilente a blanda y esponjosa (2).

El diagnóstico diferencial debe establecerse con el resto de lesiones exofíticas de los tejidos blandos bucales (Tabla 1).

La histopatología de la hiperplasia fibrosa se caracteriza fundamentalmente por la presencia de proliferación de fibroblastos y fibras de colágena, rodeadas por una capa de epitelio escamoso estratificado delgado con crestas poco profundas (3).

El tratamiento de esta tumoración, consiste en la extirpación quirúrgica y la eliminación de los factores irritantes locales. Presentamos un caso de nódulo fibroso localizado en la mucosa yugal izquierda, a nivel de la carúncula de desembocadura del conduc- to de la glándula parótida izquierda. El objetivo de este trabajo es describir la técnica quirúrgica empleada para extirpar el tumor preservando la integridad del conducto de Estenon.

\section{CASO CLÍNICO}

Hombre de 57 años de edad, sin antecedentes médicos de interés, que presentaba una tumoración asintomática de nueve años de evolución a nivel de la mucosa yugal izquierda, coincidiendo con el área edéntula del segundo molar superior. El crecimiento de la lesión fue lento y en ningún momento presentó ulceraciones. La exploración intraoral mostró una masa exofítica de aspecto nodular y bien delimitada, de 2 X $2 \mathrm{~cm}$ de diámetro localizada en el tercio medio de la mucosa yugal izquierda que incluía en su interior la carúncula de desembocadura del conducto de Estenon; la tumoración presentaba un color rosa pálido y una consistencia firme a la palpación (Fig. 1). Tras provocar mediante maniobras de manipulación de la glándula parótida izquierda la salida de saliva, comprobamos que el conducto era permeable y la saliva limpia y fluida.

El tratamiento consistió en la localización y canalización del conducto de Estenon a través de su carúncula, mediante un catéter intravenoso pediátrico (Fig. 2), seguida de la biopsia-extirpación de la masa tumoral con bisturí frío, respetando tanto el trayecto del conducto como su carúncula de drenaje. A continuación, dejamos un drenaje activo intraoral a través de la carúncula (Fig. 3) durante una semana y aplicamos presión externa en la glándula parótida cada 48 horas.

El examen histopatológico mostró un tejido fibroso colagenizado orientado en haces en todos los planos del espacio, sin atipias y revestido por epitelio plano estratificado, compatible con una hiperplasia fibrosa (Fig. 4 y 5). 
Camacho Alonso F, Saura Inglés A, López Jornet P, Bermejo Fenoll A

Tratamiento quirúrgico de una hiperplasia fibrosa localizada en la desembocadura del conducto de Estenon.

Descripción de la técnica

TABLA I. LESIONES EXOFÍTICAS DE LOS TEJIDOS BLANDOS BUCALES

\begin{tabular}{|c|c|c|c|c|c|c|}
\hline $\begin{array}{l}\text { Hiperplasias y } \\
\text { tumores benignos }\end{array}$ & $\begin{array}{c}\text { Tejido del } \\
\text { que derivan }\end{array}$ & $\begin{array}{l}\text { Localización } \\
\text { mas frecuente }\end{array}$ & Aspécto clínico & Sintomatología & Etiología & Histopatología \\
\hline $\begin{array}{l}\text { Hiperplasia } \\
\text { fibrosa }\end{array}$ & Conectivo & $\begin{array}{c}\text { - Mucosa yugal } \\
\text { - Encía } \\
\text { - Lengua } \\
\text { - Paladar blando }\end{array}$ & $\begin{array}{l}\text { - Nodular } \\
\text { - Elevado } \\
\text { - Pedunculado } \\
\text { - Sésil }\end{array}$ & Asintomático & $\begin{array}{l}\text { Posible origen } \\
\text { traumático }\end{array}$ & $\begin{array}{l}\text { - Proliferación de } \\
\text { fibroblastos y fibras de } \\
\text { colágena. } \\
\text { - Cubierta por una capa } \\
\text { de epitelio estratificado }\end{array}$ \\
\hline $\begin{array}{l}\text { Papiloma de } \\
\text { células } \\
\text { escamosas }\end{array}$ & Epitelial & $\begin{array}{l}\text { - Lengua } \\
\text { - Paladar } \\
\text { - Mucosa yugal } \\
\text { - Encía } \\
\text { - Labios }\end{array}$ & $\begin{array}{c}\text { - Masa exofítica } \\
\text { - Proyecciones } \\
\text { dactiloformes } \\
\text { - Aspecto verrugoso } \\
\text { o filiforme }\end{array}$ & Asintomático & $\begin{array}{c}\text { - Desconocida } \\
\text { Posible relación con } \\
\text { el virus del } \\
\text { papiloma humano }\end{array}$ & Hiperortoqueratosis \\
\hline Lipoma & Adiposo & $\begin{array}{l}\text { - Lengua } \\
\text { - Suelo de boca } \\
\text { - Mucosa yugal } \\
\text { - Labio }\end{array}$ & $\begin{array}{c}\text { - Masa esférica } \\
\text { - Superficie lisa } \\
\text { - Sésil } \\
\text { - Pediculado } \\
\text { - Color rosa claro o } \\
\text { amarillento } \\
\text { - Consistencia blanda } \\
\text { o semifirme }\end{array}$ & Asintomático & Desconocido & $\begin{array}{l}\text { Gran cantidad de } \\
\text { células adiposas } \\
\text { maduras en una } \\
\text { matriz de tejido } \\
\text { fibroso }\end{array}$ \\
\hline Leiomioma & $\begin{array}{l}\text { Muscular } \\
\text { (liso) }\end{array}$ & $\begin{array}{l}\text { - Lengua } \\
\text { - Paladar } \\
\text { - Mucosa yugal } \\
\text { - Encía } \\
\text { - Labio }\end{array}$ & $\begin{array}{c}\text { - Pequeño tamaño } \\
\text { - Superficial } \\
\text { - Pediculado } \\
\text { - Crecimiento lento } \\
\text { - Cosistencia duro- } \\
\text { elástica } \\
\text { - Color violáceo }\end{array}$ & Asintomático & Desconocido & $\begin{array}{c}\text { Proliferación } \\
\text { compacta de fibras } \\
\text { musculares que rodean } \\
\text { luces vasculares }\end{array}$ \\
\hline Rabdomioma & $\begin{array}{l}\text { Muscular } \\
\text { (estriado) }\end{array}$ & $\begin{array}{l}\text { Infrecuente en la } \\
\text { cavidad oral }\end{array}$ & $\begin{array}{c}\text { - Nódulo bien } \\
\text { circunscrito } \\
\text { - Crecimiento lento }\end{array}$ & Asintomático & Desconocido & $\begin{array}{c}\text { Proliferaciones celulares } \\
\text { similares a las del } \\
\text { músculo estriado } \\
\text { - Es necesario: } \\
\text { inmunohistoquímica }\end{array}$ \\
\hline Neuroma & Nervioso & $\begin{array}{l}\text { - Mandibula } \\
\text { - Labio inferior } \\
\text { - Lengua }\end{array}$ & $\begin{array}{c}\text { Nódulo delimitado } \\
\text { que sobresale de la } \\
\text { mucosa }\end{array}$ & Doloroso & $\begin{array}{c}\text { Reacción } \\
\text { reparativa al } \\
\text { cortarse un nervio }\end{array}$ & $\begin{array}{c}\text { Masa irregular de axones } \\
\text { y vainas nerviosas } \\
\text { separadas por tejido } \\
\text { conectivo fibroso }\end{array}$ \\
\hline Neurilemona & $\begin{array}{l}\text { Células de } \\
\text { Schwann }\end{array}$ & $\begin{array}{l}\text { Próxima a } \\
\text { nervios }\end{array}$ & $\begin{array}{l}\text { Nódulo único o } \\
\text { múltiples en neuro- } \\
\text { fibromatosis }\end{array}$ & Asintomático & $\begin{array}{l}\text { Posible origen } \\
\text { traumático }\end{array}$ & $\begin{array}{l}\text { Proliferación encap- } \\
\text { sulada con elementos } \\
\text { celulares fusiformes }\end{array}$ \\
\hline Neurofibroma & Nervioso & $\begin{array}{c}\text { Lengua } \\
\text { (principalmente) }\end{array}$ & $\begin{array}{l}\text { Nódulo único o } \\
\text { múltiples en neuro- } \\
\text { fibromatosis }\end{array}$ & Asintomático & Desconocido & $\begin{array}{c}\text { Elementos comunes al } \\
\text { neuroma y al fibroma }\end{array}$ \\
\hline Hemangioma & $\begin{array}{c}\text { Vasos } \\
\text { sanguíneos }\end{array}$ & $\begin{array}{c}\text { Cualquier } \\
\text { localización oral }\end{array}$ & $\begin{array}{l}\text { Nódulo unico de } \\
\text { color rojo vino } \\
\text { brillante y extendido }\end{array}$ & Asintomático & $\begin{array}{c}\text { Posible relación con } \\
\text { el factor de } \\
\text { crecimiento de } \\
\text { fibroblastos }\end{array}$ & $\begin{array}{l}\text { Multiplicación de } \\
\text { células endoteliales }\end{array}$ \\
\hline Linfangioma & $\begin{array}{l}\text { Vasos } \\
\text { linfáticos }\end{array}$ & $\begin{array}{l}\text { - Lengua. } \\
\text { - Paladar blando. } \\
\text { - Labios. } \\
\text { - Mucosa yugal }\end{array}$ & $\begin{array}{l}\text { Nódulo uni o bilate- } \\
\text { ral de tamaño } \\
\text { variable }\end{array}$ & Asintomático & Desconocido & $\begin{array}{c}\text { Vasos linfáticos } \\
\text { dilatados y revestidos } \\
\text { por células } \\
\text { endoteliales }\end{array}$ \\
\hline
\end{tabular}




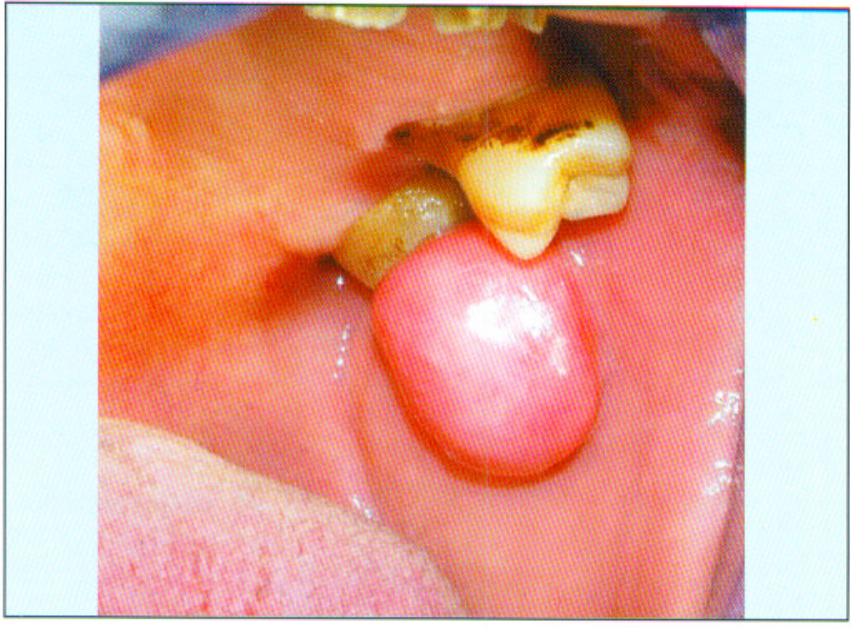

Fig. 1. Imagen clínica intraoral que muestra la localización de la lesión exofítica a nivel de la desembocadura del conducto de Estenon.

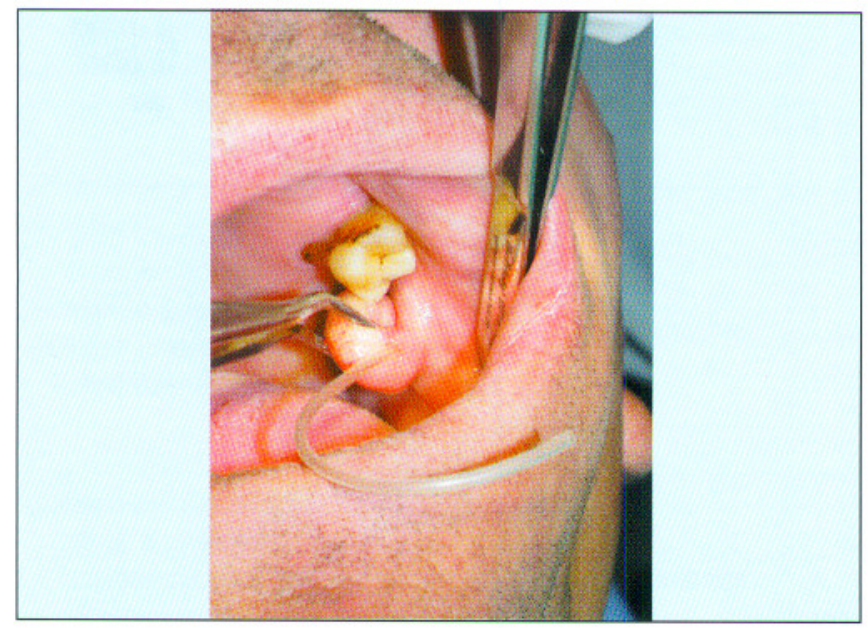

Fig. 2. Identificación y canalización del conducto de Estenon a través de su carúncula de desembocadura.

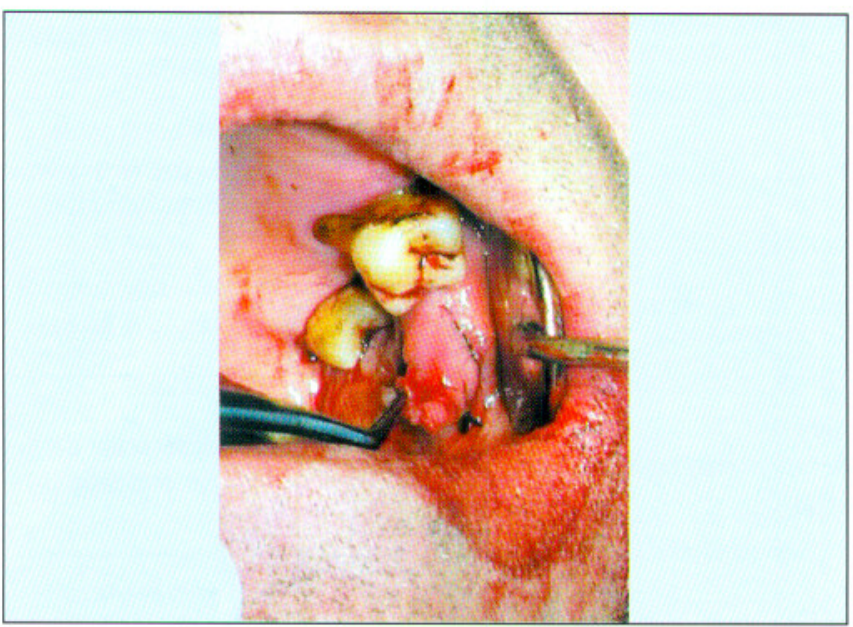

Fig. 3. Detalle de la porción externa del drenaje activo extraoral colocado tras la extirpación del tumor.

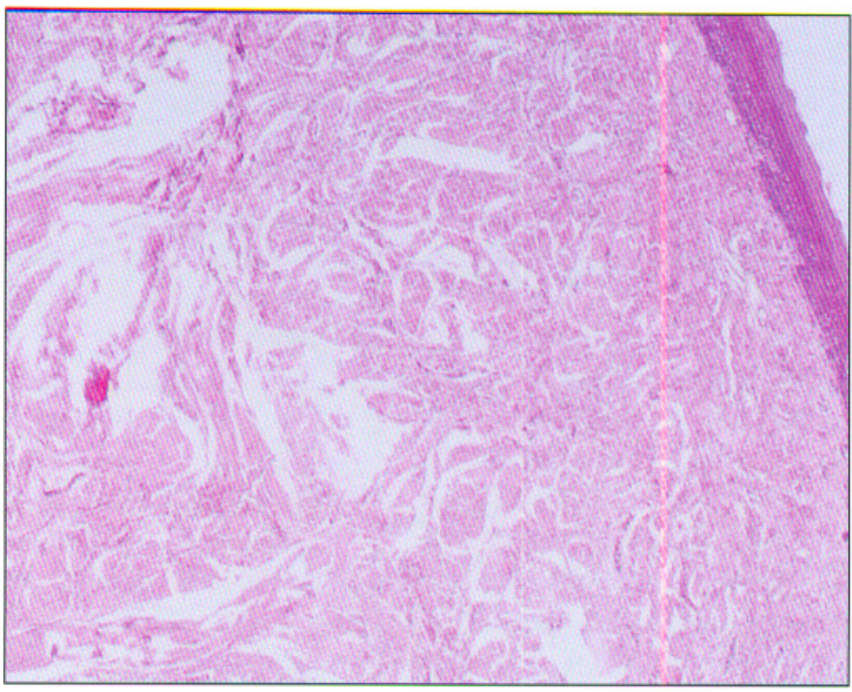

Fig. 4. Detalle del estudio histopatológico, donde se observa un tejido fibroso con haces de colágeno, revestido por un epitelio plano estratificado (Tinción con hematoxilina-eosina, X 200).

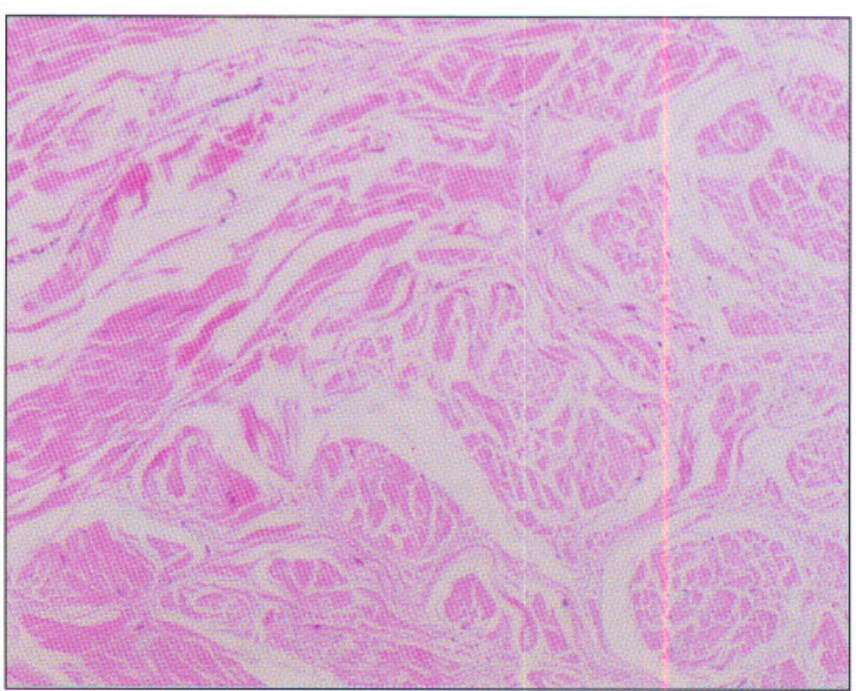

Fig. 5. Imagen de la muestra anterior donde se observa a mayor aumento el tejido fibroso (Tinción con hematoxilina-eosina, X 400).

Un mes después de la cirugía se rehabilitó el maxilar superior del paciente mediante una prótesis removible de metal-resina. Tras un año de evolución, no han aparecido recidivas y el drenaje de la glándula parótida izquierda es normal.

\section{DISCUSIÓN}

Hemos presentado la técnica quirúrgica empleada en el tratamiento de una hiperplasia fibrosa locali- 
zada a nivel de la desembocadura del conducto de Estenon. Lewkowicz y cols (4) también describen la canalización del conducto durante la reparación de traumatismos localizados en la región parotídea, mediante un catéter intravenoso pediátrico. Esto permite localizar el trayecto del conducto y evitar desgarros del mismo. En caso de producirse una laceración del conducto, si el defecto es inferior a $1 \mathrm{~cm}$, Van Sickels y Alexander (5) recomiendan la sutura de ambos extremos mediante nylon (de 9-0 o 10-0) o seda (de 7-0 u 8-0) y mantener el catéter durante dos semanas. Algunos autores (6) en estos casos prefieren aproximar los extremos del conducto con grapas microvasculares de baja tensión. Cuando el defecto es mayor de $1 \mathrm{~cm}$, la anastomosis directa es imposible, por lo que algunos autores (7) han ensayado en animales el injerto venoso autógeno, obteniendo buenos resultados. En extensas laceraciones del conducto donde este está totalmente desgarrado y no hay posibilidad de unir los dos extremos, está indicado clampar el extremo más próximo a la glándula (4). Epker y Bournette (8) sugieren además la apertura de un drenaje extraoral para prevenir la formación de un mucocele.

En nuestro caso, una vez extirpado el nódulo fibroso, dejamos un drenaje activo intraoral durante una semana para evitar el cierre de la carúncula y/o la estenosis del conducto de Estenon durante el proceso de cicatrización. Además realizamos una estimulación activa de la glándula parótida cada 48 horas. Si en alguna de estas estimulaciones, la parótida no segrega saliva con normalidad, está indicada (8) la aspiración de la misma a través de la cánula, evitando así su acúmulo.

En caso de que el drenaje no fuese efectivo y se produjera una estenosis del Estenon, Kim y cols (9) describen un tratamiento basado en la angioplastia con un pequeño globo (catéter de $3 \mathrm{~mm}$ usado en cirugía cardíaca), introducido hasta la zona de la estenosis con un alambre flexible de $0,35 \mathrm{~mm}$, según la técnica de Roberts y cols (10).

Finalmente, el espacio edéntulo de la zona donde se localizaba la lesión fue rehabilitado tras la cicatrización de la herida, para evitar recidibas atribuibles a la etiología diapnéusica por succión $(1,2)$.

\section{BIBLIOGRAFÍA}

1. López Jornet P. Hiperplasias y tumores benignos de los tejidos blandos bucales. En: Bermejo Fenoll A, eds. Medicina Bucal. Enfermedades mucocutáneas y de las glándulas salivales. Madrid: Editorial Síntesis; 1998.p.231-46.

2. Bagán JV, Vera. Patología de la mucosa oral. 1a ed. Barcelona: Syntex Latino SA. 1989.p.59-68.

3. Christopoulos P, Sklavounou A, Patrikiou A. True fibroma of the oral mucosa: a case report. Int $\mathrm{J}$ Oral Maxillofac Surg 1994; 23:98-9.

4. Lewkowicz AA, Hasson O, Nahlieli O. Traumatic injuries to the parotid gland and duct. J Oral Maxillofac Surg 2002; 60:676-80.

5. Van Sickels JE. Parotid duct injuries. Oral Surg Oral Med Oral Pathol 1981:364-7.

6. Haller JR. Trauma to the salivary glands. Otolaryngol Clin North Am 1999; 32:907-18.

7. Chudakov O, Ludchik T. Microsurgical repair of Stensen's \& Wharton's ducts with autogenous venous grafts. An experimental study on dogs. Int J Oral Maxillofac Surg 1999; 28:70-3.

8. Epker BN, Burnette JC. Trauma to the parotid gland and duct: primary treatment and management of complications. J Oral Surg 1970; 28:657-70.

9. Gear KJ, Hay KD, Stumpel J. Treatment of parotid ductal stenosis and concomitant resolution of autonomic symptomatology. Oral Surg Oral Med Oral Pathol Oral Radiol Endod 2002; 94:632-5.

10. Roberts DN, Juman S, Hall JR, Jonathan DA. Parotid duct stenosis: interventional radiology to the rescue. Ann R Coll Surg Engl 1995; 77:444-6.

\section{CORRESPONDENCIA}

Fabio Camacho Alonso

Clinica Odontologica Universitaria

Hospital Morales Meseguer

Adv. Marques de los velez $\mathrm{s} / \mathrm{n}$

Murcia 30008

Email: fabiosurgery@hotmail.com 